\title{
Differentially Private Optimal Transport: Application to Domain Adaptation
}

\author{
Nam LeTien ${ }^{1}$, Amaury Habrard ${ }^{1}$ and Marc Sebban ${ }^{1}$ \\ ${ }^{1}$ Univ Lyon, UJM-Saint-Etienne, CNRS, Institut d'Optique Graduate School, Laboratoire Hubert Curien \\ UMR 5516, F-42023, SAINT-ETIENNE, France \\ \{tien.le, amaury.habrard, marc.sebban\}@univ-st-etienne.fr
}

\begin{abstract}
Optimal transport has received much attention during the past few years to deal with domain adaptation tasks. The goal is to transfer knowledge from a source domain to a target domain by finding a transportation of minimal cost moving the source distribution to the target one. In this paper, we address the challenging task of privacy preserving domain adaptation by optimal transport. Using the Johnson-Lindenstrauss transform together with some noise, we present the first differentially private optimal transport model and show how it can be directly applied on both unsupervised and semi-supervised domain adaptation scenarios. Our theoretically grounded method allows the optimization of the transportation plan and the Wasserstein distance between the two distributions while protecting the data of both domains. We perform an extensive series of experiments on various benchmarks (VisDA, Office-Home and Office-Caltech datasets) that demonstrates the efficiency of our method compared to non-private strategies.
\end{abstract}

\section{Introduction}

Optimal Transport (OT) [Villani, 2008] is a geometric theory that allows us to put a distance (e.g. Wasserstein distance, earth mover distance) on the space of probability measures. As probability measures occur in many scenarios in machine learning, OT has received a lot of attention during the past few years in this community [Cuturi, 2013; Frogner et al., 2015; Arjovsky et al., 2017]. For instance, probability measures take the form of color histograms when comparing images, bags of words in document analysis, activation maps in brain imaging, etc. Perhaps, the most exciting recent application of OT in machine learning comes from the wide use of generative models and the need of some notion of distance to measure the divergence between generated data and the actual generative model. Very recently, OT has been shown to be a useful and intuitive tool to address domain adaptation tasks [Courty et al., 2017b; Courty et al., 2017a; Shen et al., 2018], where the goal is to transfer knowledge from a source domain to a target domain. In such a scenario, regularized OT aims at finding a transportation of minimal cost aligning labeled source data on unlabeled target examples. Then a classifier can be learned from the source examples and deployed on the target domain. OT for domain adaptation has also received recent attention from a theoretical perspective. In [Redko et al., 2017], the authors show how the Wasserstein distance can be used to derive generalization bounds on the target error. All things considered, OT can be seen nowadays as a theoretically grounded competitive framework to perform transfer learning.

Optimizing the transportation that aligns the source and target distributions boils down to finding a coupling matrix that moves the source data to the target ones and allows us to induce the Wasserstein distance. Therefore, this procedure requires to share data from both sources at some point of the learning process that may appear to be an unacceptable solution when source and/or target data contain sensitive information (e.g. about the medical history of patients). The contributions of this paper lie in the setting of differentially private OT and its application to Domain Adaptation. Differential privacy [Dwork et al., 2006] provides a strong theoretical-grounded guarantee for the privacy of individuals against attackers and has become the standard for formal privacy in machine learning. The basic idea of differential privacy is to introduce randomness in the communication that preserves privacy even against an adversary possessing arbitrary side information and having access to the communication. It turns out that there has been a rich amount of work on differentially private machine learning, such as in logistic regression [Chaudhuri and Monteleoni, 2009], principal component analysis [Hardt and Roth, 2013], boosting [Dwork et al., 2010], support vector machines [Rubinstein et al., 2009], or more recently on deep learning [Abadi et al., 2016; Shokri and Shmatikov, 2015] and semi-supervised deep learning [Papernot et al., 2017]. However, it is worth noting that privacy preserving domain adaptation is surprisingly under-developed. Recently, [Wang et al., 2018] and [Guo et al., 2018] proposed two similar methods, combining hypothesis transfer learning with private logistic regression. However, the first model needs to have access to a publicly available auxiliary dataset as a bridge to transfer knowledge from the source to the target, while the second trains on a fully labeled target data; both are strong constraints that do not hold in the standard (both unsupervised and semi-supervised) domain adaptation setting. 
The objective of this paper is to fill the gap mentioned above and address the challenging privacy preserving problem when OT is used to transfer knowledge from a source to a target. To do so, we present two new algorithms. The first one, called Differentially Private Optimal Transport (DPOT), makes use of the Johnson-Lindenstrauss transform [Johnson and Lindenstrauss, 1984] which linearly projects a set of examples onto a small feature space by a random matrix that preserves the pairwise distances. Adapted to the context of OT, DPOT allows us to jointly compute the coupling matrix and the Wasserstein distance between two domains under the differential privacy constraint, by adding noise on the random projection. As far as we know, DPOT is the first differentially private optimal transport algorithm. Experimental results show that DPOT preserves the Wasserstein distance on the space of probability measures compared to a non private OT approach. We then build upon DPOT the first complete differentially private domain adaptation model (DPDA) where the learner can benefit from labeled source data to improve a different but related target task while still ensuring the privacy of each source of data. We provide theoretical guaranty of our method and demonstrate its efficiency empirically on various benchmarks.

The rest of this paper is organized as follows: Section 2 presents the required background in Optimal Transport, Domain Adaptation and Differential Privacy. In Section 3, based on the Johnson-Lindenstrauss transform, we introduce DPOT. Section 4 is devoted to the presentation of DPDA, the first Differentially Private Domain Adaptation algorithm based on Optimal Transport for which differential privacy guarantees are derived. In Section 5, we first show that DPOT allows us to efficiently approximate the true Wasserstein distance that would be obtained without privacy constraint. Then, we report the results of an extensive experimental study performed on various benchmarks (VisDA, Office-Home and OfficeCaltech datasets) that demonstrate the efficiency of DPDA compared to non-private strategies.

\section{Background and Related Work}

\subsection{Optimal Transport}

Let $\mu_{1}$ and $\mu_{2}$ be two probability measures. Given some cost function $c$, optimal transport (OT) seeks a transportation plan of minimal cost that moves $\mu_{1}$ to $\mu_{2}$. Let $\Pi$ be the space of joint probability distributions with marginals $\mu_{1}$ and $\mu_{2}$. In the relaxed formulation of Kantorovitch [1942], the optimal transportation takes the form of a distribution (or coupling) $\gamma_{0} \in \Pi$ that minimizes the following quantity:

$$
W\left(\mu_{1}, \mu_{2}\right)=\min _{\gamma \in \Pi} \int_{\Omega_{s} \times \Omega_{t}} c\left(x^{s} x^{t}\right) d \gamma\left(x^{s}, x^{t}\right),
$$

where $c\left(x^{s}, x^{t}\right)$ is this paper the Euclidean distance between $x^{s} \in \Omega_{s}$ and $x^{t} \in \Omega_{t}$. The quantity $W\left(\mu_{1}, \mu_{2}\right)$ is called the Wasserstein distance between the two distributions $\mu_{1}$ and $\mu_{2}$. When $\mu_{1}, \mu_{2}$ are defined as empirical measures between two datasets $X_{s}$ and $X_{t}$, then

$$
\gamma_{0}=\underset{\gamma \in \Pi}{\operatorname{argmin}}\langle\gamma, C\rangle_{F},
$$

where $\langle.,\rangle_{F}$ is the Frobenius dot product and $C$ is the cost matrix between $X_{s}$ and $X_{t}$. Then, the Wasserstein distance between $X_{s}$ and $X_{t}$ is $W\left(X_{s}, X_{t}\right)=\left\langle\gamma_{0}, C\right\rangle_{F}$.

\subsection{Domain Adaptation}

Domain adaptation [Pan and Yang, 2010] aims at using available labeled data from a source domain (e.g. synthetic data) to facilitate the learning process in a target domain (e.g. real data) with a different underlying distribution. In unsupervised domain adaptation, we assume to have access to only unlabeled target data, while in semi-supervised tasks, a small amount of supervision is available, but not large enough to learn well only from the labeled target data. Most recent state-of-the-art methods either learn a common latent feature space between the two domains, e.g. DANN [Ganin et al., 2016], or map the samples of one domain to the other, e.g. ADDA [Tzeng et al., 2017].

Optimal transport was recently applied successfully to domain adaptation, where the Wasserstein distance is used as a divergence measure between the two domains that a domain adaptation algorithm aims at minimizing. In [Courty et al., 2017b], the source samples are transported to the target domain using the coupling matrix $\gamma_{0}$. In [Courty et al., 2017a], the authors optimize both the coupling matrix and a target prediction function, while [Shen et al., 2018] minimize the domain divergence in the latent space using the WassersteinGAN [Arjovsky et al., 2017].

\subsection{Differential Privacy}

Differential Privacy was introduced by Dwork et. al. [2006] and constitutes nowadays a standard for privacy guarantees.

Definition 1 (Differential Privacy, see eg. [Abadi et al., 2016]). A randomized mechanism $\mathcal{M}: \mathcal{X}^{n} \rightarrow \mathbb{R}^{d}$ satisfies $(\varepsilon, \delta)$-differential privacy iffor any two datasets $X, X^{\prime} \in \mathcal{X}^{n}$ differing by a single element and for any set of possible output $\mathcal{O} \subseteq$ Range $(\mathcal{M})$ :

$$
\mathbb{P}(\mathcal{M}(X) \in \mathcal{O}) \leq e^{\varepsilon} \mathbb{P}\left(\mathcal{M}\left(X^{\prime}\right) \in \mathcal{O}\right)+\delta
$$

There has been a rich amount of work on differentially private machine learning, such as in logistic regression [Chaudhuri and Monteleoni, 2009], principal component analysis [Hardt and Roth, 2013], boosting [Dwork et al., 2010] and support vector machine [Rubinstein et al., 2009]. Private deep learning methods were also introduced recently; [Abadi et al., 2016] provided a differentially private SGD method for training a deep neural network, where at each step, the gradient is clipped and some noise is added to protect privacy. On the other hand, [Shokri and Shmatikov, 2015] proposed a distributed selective SGD: several models are trained independently using SGD and they selectively upload the gradients to a global one. Similarly, [Papernot et al., 2017] train several teachers with private data, then predict pseudo-labels via a majority vote for unlabeled public data that are used by a student to train.

It is worth noticing that privacy preserving domain adaptation has not received much attention. Deep learning methods such as [Abadi et al., 2016; Papernot et al., 2017] are 
designed for standard classification tasks with the assumption that all data are drawn from the same underlying distribution, which is not the case in domain adaption (although technically, these algorithms can be deployed out of the box for domain adaptation through training a source-only model) Private multiparty learning methods, such as [Pathak et al., 2010; Hamm et al., 2016] could also be applied to semisupervised domain adaptation, but they face two drawbacks: (1) the amount of target labeled data is usually very limited for a meaningful aggregation, and (2) these models assume that the domain distributions are identical and so ignore the domain shift. As already mentioned, [Wang et al., 2018] and [Guo et al., 2018] proposed two methods that combine hypothesis transfer learning with private logistic regression. However, the first model needs to have access to a publicly available auxiliary dataset as a bridge to transfer knowledge from the source to the target, while the second trains on a fully labeled target data; both are strong constraints that do not hold in the standard (both unsupervised and semi-supervised) domain adaptation setting.

\subsection{Johnson-Lindenstrauss Transform}

Let $\mathcal{N}(0, \sigma)^{k \times \ell}$ be a $k \times \ell$ matrix where each entry is drawn i.i.d. from $\mathcal{N}(0, \sigma)$. A variant of the famous JohnsonLindenstrauss Lemma [Johnson and Lindenstrauss, 1984] states that a linear transformation of a set of $n$ data points to a much smaller subspace by such a random matrix has a high probability to retain the pairwise distances within $(1 \pm \eta)$ factor for some $\eta \in[0,0.5]$ (see eg. [Blocki et al., 2012]).

Theorem 1. Let $v_{1}, \ldots, v_{n}$ be a set of $n$ points in $\mathbb{R}^{k}$. For any $\eta, \ell>0$ and an $\mathcal{N}\left(0, \frac{1}{\ell}\right)^{k \times \ell}$ matrix $M$, with probability at least $1-\frac{2}{\exp \left(\ell \eta^{2} / 8\right)}$ the following holds for every $i, j$ :

$$
1-\eta \leq \frac{\left\|v_{i}^{T} M-v_{j}^{T} M\right\|_{2}^{2}}{\left\|v_{i}-v_{j}\right\|_{2}^{2}} \leq 1+\eta .
$$

The Johnson-Lindenstrauss transform is widely used across many areas of computer science such as computational speedups, machine learning, information retrieval. The interested reader may find an extensive overview of the topic in the monograph written by Vempala [2005]. Due to its randomness, the Johnson-Lindenstrauss transform was also used in differential privacy. [Blocki et al., 2012] showed that it preserves the differential privacy, as long as all singular values of the database are sufficiently large. [Kenthapadi et al., 2013] showed that by simply applying the Johnson-Lindenstrauss transform together with some noise, one can publish the pairwise distances of elements while protecting the privacy of the original data.

As OT resorts to a pairwise distance matrix to find the optimal coupling and the Wasserstein distance, we suggest in the next sections to benefit from the Johnson-Lindenstrauss transform and the idea introduced in [Kenthapadi et al., 2013] to build the first differentially private OT method (DPOT); then, we use it to design a new privacy-preserving domain adaptation algorithm (DPDA).

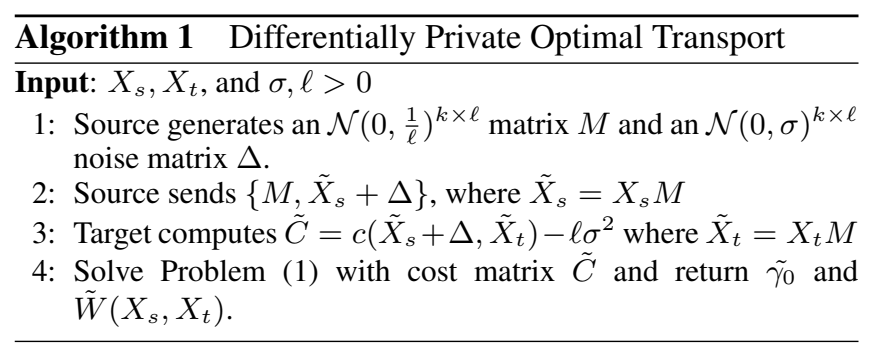

\section{Differentially Private Optimal Transport}

Let $X_{s}$ and $X_{t}$ be a source and a target dataset of size $n_{s} \times k$ and $n_{t} \times k$ (where $k$ is the number of features) respectively. A standard OT procedure aims at finding the Wasserstein distance $W$ and the empirical transportation plan $\gamma_{0}$ between $X_{s}$ and $X_{t}$.

Usually, one has access to both $X_{s}$ and $X_{t}$ to calculate the cost matrix $C$, typically as the matrix of pairwise Euclidean distances $c\left(X_{s}, X_{t}\right)$ between samples of the source and target sets. Then we solve the optimization Problem (1) to get $\gamma_{0}$ and $W$. However, under privacy constraints, source and target parties may not be willing to release their data, making optimal transport a challenging task. To overcome this problem, let us use the result proved in Kenthapadi et al. [2013] stating that by applying the Johnson-Lindenstrauss transform with some additional Gaussian noise, one can publish the pairwise distances of elements privately (see Kenthapadi et al. [2013] for more details).

We exploit this idea in the context of OT where the objective is to align $X_{s}$ on $X_{t}$. Therefore, the data we want to publish safely here is the source set $X_{s}$. Algorithm 1 describes the pseudo-code of our Differentially Private Optimal Transport (DPOT) algorithm. The main steps of DPOT are the following: In Step 1, we generate a random matrix $M$ according to [Johnson and Lindenstrauss, 1984] and a noise matrix $\Delta$ according to [Kenthapadi et al., 2013]. Step 2 boils down to publishing both $M$ and the source data $\tilde{X}_{s}+\Delta$ after a random projection of $X_{s}$ by $M$ in a subspace of size $\ell$ and the addition of the noise $\Delta$. To benefit from the privacy guarantees of [Kenthapadi et al., 2013] over the pairwise (source/target) distances, one compute the distance matrix $\tilde{C}$ in Step 3 between the published source data $\tilde{X}_{s}+\Delta$ and the projected target examples $\tilde{X}_{t}=X_{t} M$. Note that $\ell \sigma^{2}$ is subtracted from each entry to cancel the bias caused by $\Delta$ (indeed, $\sigma^{2}$ is known by the target party but not $\Delta$ ). Step 4 is devoted to the resolution of Problem (1).

Kenthapadi et al. [2013], Theorem 1, showed that the mechanism of publishing $M$ and the noisy data $\tilde{X}_{s}+\Delta$ satisfies the $(\epsilon, \delta)$-differential privacy of Definition 1, for any $\varepsilon, \delta>0$ and $\sigma \geq w \frac{\sqrt{2\left(\ln \left(\frac{1}{2 \delta}\right)+\varepsilon\right)}}{\varepsilon}$ where $w=$ $\max _{1 \leq i \leq k}\left(\sum_{j=1}^{\ell} M_{i j}^{2}\right)^{\frac{1}{2}}$, that is the $\ell_{2}$-norm sensitivity of $M$, which is tightly concentrated around 1 [Kenthapadi et al., 2013]. Therefore, Algorithm 1 comes directly with the following privacy guarantee.

Theorem 2. Algorithm 1 is $(\varepsilon, \delta)$-differentially private for any $\varepsilon, \delta>0$ and $\sigma \geq w \frac{\sqrt{2\left(\ln \left(\frac{1}{2 \delta}\right)+\varepsilon\right)}}{\varepsilon}$. 


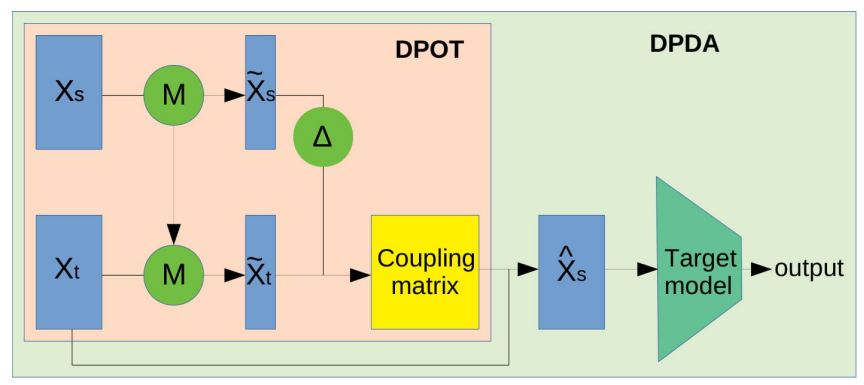

Figure 1: The architecture of DPOT and DPDA.

In Section 5, we will report experimental results showing that by satisfying the previous constraint on the noise $\sigma$, the Wasserstein distance $\tilde{W}\left(X_{s}, X_{t}\right)$ computed by Algorithm 1 remains very close to the true $W\left(X_{s}, X_{t}\right)$, while preserving the privacy of the data. Therefore, we can now benefit from $\tilde{W}\left(X_{s}, X_{t}\right)$ and $\tilde{\gamma}_{0}$ to transfer knowledge from a source domain to a target one to deal with domain adaptation in a differential privacy setting.

\section{Differentially Private Domain Adaptation}

Extending DPOT for domain adaptation requires (i) to transport the source data to the target domain thanks to a private barycentric mapping, (ii) to transfer the source labels while ensuring privacy, (iii) to add some appropriate domain adaptation regularizers for computing the coupling matrix.

Barycentric mapping. Once the coupling matrix $\tilde{\gamma_{0}}$ is computed, the source samples can be moved to the target domain using the geodesics of the Wasserstein metric. The barycentric mapping [Reich, 2013] from each source sample $x_{i}^{s} \in X_{s}$ to its corresponding image $\widehat{x}_{i}^{s}$ in the target domain is as follows

$$
\widehat{x}_{i}^{s}=\underset{x \in \mathbb{R}^{k}}{\operatorname{argmin}} \sum_{j} \tilde{\gamma}_{0}[i, j] c\left(x, x_{j}^{t}\right) .
$$

The barycentric image $\widehat{x}_{i}^{s}$ can be understood as the empirical optimal location on the target domain to transport $x_{i}^{s}$ w.r.t. the coupling matrix $\tilde{\gamma}_{0}$. Let $\widehat{X}_{s}$ be the set of barycentric images of the source data. It is now possible to train a model from the transported labeled source data $\left(\widehat{X}_{s}, Y_{s}\right)$ and use it in the target domain [Courty et al., 2017b].

Private barycentric mapping. Generally in domain adaptation, the target agent must have access to the labeled data from the source. However, under privacy constraints, this is not possible anymore. Fortunately, by combining DPOT algorithm with the barycentric mapping, we can privately transport the source data in the target domain. Indeed, as shown in Equation 2, only $\tilde{\gamma}_{0}$ and $X_{t}$ are involved in the calculation of the barycentric mapping which does not depend on the source data. The private barycentric images can be conveniently rewritten as $\widehat{X}_{s}=n_{s} \tilde{\gamma}_{0} X_{t}$ as shown in [Perrot $e t$ $a l ., 2016]$ in a non private scenario.

Transmitting source labels. To perform domain adaptation in both unsupervised and semi-supervised settings, the target party needs to have access to the source labels $Y_{s}$. To enhance the privacy of our domain adaptation algorithm, we also aim at adding some noise on the labels. To do so, we follow the principle of Histogram Queries mentioned in [Dwork et al., 2014]: The source first reorders its data $\left(X_{s}, Y_{s}\right)$ such that all samples of label 1 appear first, then samples of label 2 and so on. Hence $Y_{s}$ is now equivalent to a vector $v\left(Y_{s}\right)$ of length $q$ counting the number of samples for each label, where $q$ is the number of labels. The source party can now safely publish a noisy version of $v\left(Y_{s}\right)$ as a histogram query by adding a Laplacian noise $\operatorname{Lap}\left(\frac{1}{\varepsilon^{\prime}}\right)^{q}$, which is $\left(\varepsilon^{\prime}, 0\right)$ differentially private [Dwork et al., 2014].

Regularization techniques. There are various techniques in the literature to regularize the coupling matrix for domain adaptation tasks. In our method, we utilize two of them. The first one is the entropic regularization [Cuturi, 2013] that allows us to transform Problem 1 into a strictly convex problem and is defined as follows:

$$
R_{e}(\gamma)=-\sum_{i, j} \gamma_{i j}\left(\log \gamma_{i j}-1\right)
$$

The second one is the group Lasso regularization [Courty et al., 2017b],

$$
R_{g}(\gamma)=\sum_{j} \sum_{s}\left\|\gamma\left(I_{s}, j\right)\right\|_{2}
$$

where $\|\cdot\|_{2}$ is the $\ell_{2}$-norm, $I_{s}$ contains the indices of rows in $\gamma$ related to source samples associated to label $s, \gamma\left(I_{s}, j\right)$ is then a vector containing the coefficients of the $j^{\text {th }}$ column of $\gamma$ associated to label $s$. This regularization encourages a coupling where a given target sample receives masses from source samples having the same labels. Thus, the coupling matrix is now computed by the following equation:

$$
\tilde{\gamma}_{0}=\underset{\gamma \in \Pi}{\operatorname{argmin}}\langle\gamma, \tilde{C}\rangle_{F}+\lambda_{e} R_{e}(\gamma)+\lambda_{g} R_{g}(\gamma)
$$

where $\lambda_{s}, \lambda_{c}$ are hyper-parameters. In the semi-supervised setting, we can further exploit the available target label data by prohibiting masses from being transported between samples with different labels, which does not require any additional hyper-parameter [Courty et al., 2017b].

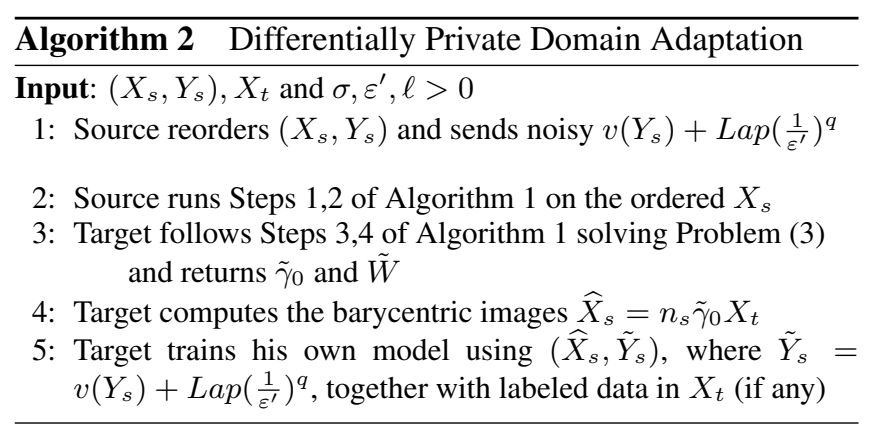

Differentially Private Domain Adaptation Algorithm. We now have all the necessary blocks for our differentially private domain adaptation algorithm DPDA, see the pseudocode in Algorithm 2, where the two parties collaboratively compute a regularized coupling matrix using Eq. (3), which 

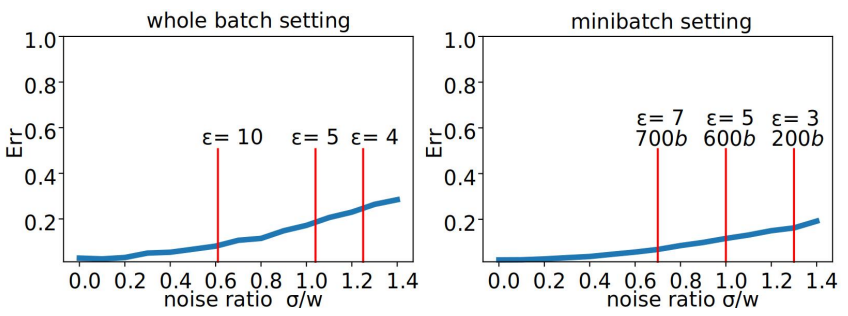

Figure 2: Evolution between noise-ratio and the accuracy of DPOT. On the right, 200b means "200 minibatches".

allows the target to obtain the barycentric images of the source data for his own training. We have shown that both mechanisms of transmitting $\left\{M, \tilde{X}_{s}+\Delta\right\}$ and the noisy vector $v\left(Y_{s}\right)$ from the source are differentially private. According to the Composition Theorem ([Dwork et al., 2014] Theorem 3.16), the composition of differentially private mechanisms is also differentially private. This means that Algorithm 2 is also differentially private.

Theorem 3. Algorithm 2 is $\left(\varepsilon+\varepsilon^{\prime}, \delta\right)$-differentially private for any $\varepsilon, \varepsilon^{\prime}, \delta>0$ and $\sigma \geq w \frac{\sqrt{2\left(\ln \left(\frac{1}{2 \delta}\right)+\varepsilon\right)}}{\varepsilon}$.

The whole workflow of our differentially private method is shown is Figure 2.

\section{Experiments}

\subsection{Private Optimal Transport with DPOT}

In this section, we empirically validate our private optimal transport method DPOT by analyzing the relation between the privacy budget $(\varepsilon, \delta)$ and the accuracy of the Wasserstein distance $\tilde{W}$ computed by DPOT. The error between $\tilde{W}$ and the true Wasserstein distance $W$ is measured here by $E r r=\left|\frac{\tilde{W}-W}{W}\right|$. We perform an experiment on the OfficeHome dataset [Venkateswara et al., 2017], using two domains with a moderate number of samples, Clipart (4365 samples) and Product (4439 samples). We follow the experimental protocol used in [Abadi et al., 2016] and [Papernot et al., 2017] by setting the privacy budget to $\delta=\frac{1}{1.2 n_{s}}$, where $n_{s}$ is the size of the source domain, and using a some moderate $\varepsilon$ with 1-digit.

Besides, it is widely known that minibatch subsampling can enhance privacy [Abadi et al., 2016; Balle et al., 2018]. Recently, [Abadi et al., 2016] introduced the privacy accountant theorem to get a tight bound on the total privacy budget $(\varepsilon, \delta)$ (i.e. the allowed leakage amount of privacy during the whole training process) when training with multibatches. In the following, we will perform experiments on two settings: whole batch and minibatch to demonstrate the effect of using minibatches on the privacy budget.

Whole batch setting. In the whole batch setting, the noiseratio $\frac{\sigma}{w}$ can be calculated directly from the privacy budget $(\varepsilon, \delta)$ by Theorem 2 . For example, when $\varepsilon=4,5,10$, we get $\frac{\sigma}{w}=1.25,1.04,0.61$, respectively. In Figure 2 (left), we plot the evolution of the error $\operatorname{Err}$ between $\tilde{W}$ and $W$ given a noise-ratio $\frac{\sigma}{w}$ on the two whole datasets (we calculate $\mathrm{Err}$ over 20 runs and report the average). Keeping in mind that we want $\varepsilon$ as small as possible, the figure shows that $E r r=0.08$ at $\varepsilon=10$ (maximum acceptable value according to the literature), while when the privacy budget is reduced to $\varepsilon=4$, the error reaches $22 \%$. In order to reduce the approximation error, the next experiment shows that using minibatches allows us to drastically reduce the privacy budget.

Minibatch setting. In the minibatch setting, the relationship between the noise-ratio $\frac{\sigma}{w}$ and the privacy budget $(\varepsilon, \delta)$ can be calculated via the privacy accountant [Abadi et al., 2016]. For $\delta=\frac{1}{1.2 n_{s}}$ and a minibatch of size 128, the noiseratio $\frac{\sigma}{w}$ depends on both $\varepsilon$ and the number of minibatches we wish to run. For example, if $\frac{\sigma}{w}=1$ then for $\varepsilon=3,5,7$ we can run 200, 600, 1200 minibatches respectively, while when $\frac{\sigma}{w}=0.7$, we can run only $4,60,200$ batches for $\varepsilon=3,5,7$. We can note in Figure 2 (right) that the error Err of DPOT (average over 1000 random minibatches), for $\frac{\sigma}{w}=0.7,1,1.3$, is $7 \%, 10 \%$ and $15 \%$, respectively. This is substantially lower than running on the whole batch. Empirically, we found that the effect of the noise-ratio $\frac{\sigma}{w}$ on the accuracy of DPOT in the minibatch setting is the same across datasets, but the privacy budget (or the number of allowed minibatches for a given budget) would be different since the size of the dataset plays an important role. For example, as the Synthetic domain of VisDA dataset [Peng et al., 2017] has 150k samples, we can run $10 \mathrm{k}$ batches for $\varepsilon=1, \frac{\sigma}{w}=1$ or $30 \mathrm{k}$ batches for $\varepsilon=2, \frac{\sigma}{w}=0.8$.

\subsection{Private Domain Adaptation with DPDA}

The previous experimental study showed that minibatches allow us to improve the quality of the outputs of DPOT with a smaller privacy budget. For this reason, the next experiments are performed only in this setting.

Benchmarks. We evaluate our method on three domain adaptation benchmarks from the classical Office-Caltech dataset [Saenko et al., 2010] to the more recent and challenging VisDA [Peng et al., 2017] and Office-Home [Venkateswara et al., 2017] datasets. The three datasets offer a wide range of scenarios: Office-Caltech is a very small dataset with 150-1000 samples per domain; VisDA contains a large collection (200k samples in total) of high quality images, while Office-Home has a moderate size (4000 samples/domain) but many (65) classes. In Office-Caltech and Office-Home, there are four different domains each, coming from different sources, while VisDA is a specific dataset for simulation-to-real adaptation, where the source domain contains 150k synthetic images rendering of 3D models from different angles and the target domain contains 50k samples of natural images. We conduct the experiments on both unsupervised and semi-supervised settings.

Baselines. Our baselines include the state-of-the-art private semi-supervised method PATE [Papernot et al., 2017], where the teachers hold source data and the student holds target data, the non-private optimal transport domain adapation method OTDA [Courty et al., 2017b] which we build our model DPDA upon, and another state-of-the-art non-private domain adaptation method ADDA [Tzeng et al., 2017] using a domain-adversarial technique. We do not add [Wang et al., 2018] and [Guo et al., 2018] as baselines since both only 
Proceedings of the Twenty-Eighth International Joint Conference on Artificial Intelligence (IJCAI-19)

\begin{tabular}{|l|cc|cc|}
\hline & PATE & DPDA & OTDA & ADDA \\
\hline $\mathrm{A} \rightarrow \mathrm{C}$ & 81.4 & 87.6 & 88.3 & 86.9 \\
$\mathrm{~A} \rightarrow \mathrm{D}$ & 88.5 & 91.0 & 94.2 & 91.7 \\
$\mathrm{~A} \rightarrow \mathrm{W}$ & 77.9 & 96.4 & 95.5 & 96.8 \\
$\mathrm{C} \rightarrow \mathrm{A}$ & 91.0 & 91.9 & 92.5 & 92.1 \\
$\mathrm{C} \rightarrow \mathrm{D}$ & 85.9 & 92.0 & 92.5 & 91.7 \\
$\mathrm{C} \rightarrow \mathrm{W}$ & 82.0 & 93.9 & 95.3 & 95.5 \\
$\mathrm{D} \rightarrow \mathrm{A}$ & 67.2 & 89.1 & 92.5 & 89.8 \\
$\mathrm{D} \rightarrow \mathrm{C}$ & 58.5 & 79.0 & 87.0 & 85.8 \\
$\mathrm{D} \rightarrow \mathrm{W}$ & 81.3 & 96.1 & 98.7 & 96.9 \\
$\mathrm{~W} \rightarrow \mathrm{A}$ & 79.2 & 93.1 & 93.2 & 93.0 \\
$\mathrm{~W} \rightarrow \mathrm{C}$ & 70.9 & 86.0 & 87.6 & 87.6 \\
$\mathrm{~W} \rightarrow \mathrm{D}$ & 98.7 & 98.0 & 98.7 & 99.2 \\
\hline Average & 80.2 & 91.2 & 93.0 & 92.3 \\
\hline
\end{tabular}

Table 1: Performance (accuracy \%) on Office-Caltech dataset in the unsupervised setting.

\begin{tabular}{|l|cc|cc|}
\hline & PATE & DPDA & OTDA & ADDA \\
\hline plane & 87.7 & 88.5 & 89.2 & 96.2 \\
bicycle & 31.3 & 66.2 & 64.8 & 71.4 \\
bus & 76.4 & 75.8 & 75.7 & 76.2 \\
car & 69.6 & 59.1 & 58.6 & 44.3 \\
horse & 86.9 & 86.5 & 87.0 & 65.8 \\
knife & 53.4 & 70.4 & 62.1 & 83.0 \\
motorcycle & 80.8 & 69.9 & 74.6 & 87.7 \\
person & 60.1 & 71.6 & 70.8 & 44.8 \\
plant & 64.7 & 75.9 & 76.3 & 81.8 \\
skateboard & 22.9 & 49.3 & 50.5 & 68.7 \\
train & 55.4 & 86.8 & 87.7 & 91.4 \\
truck & 7.0 & 39.5 & 40.9 & 40.4 \\
\hline All & 60.6 & 68.8 & 69.1 & 67.3 \\
\hline
\end{tabular}

Table 2: Classwise performance (accuracy \%) of VisDA dataset in the unsupervised setting.

work on binary-classification and the first requires an auxiliary public dataset while the second requires fully labeled target data.

For the Office-Caltech dataset, we use the common DeCAF features as input, while for VisDA and Office-Home, we use the NASNet model [Zoph et al., 2018] with weights pre-trained on ImageNet as a base model to extract features from the images. All methods are written in Keras [Chollet, 2015] with the same target model architecture (a 3-layer neural network) for fair comparison. The coupling matrices are computed using the POT library [Flamary and Courty, 2017]. As already said, we train all the models using minibatches.

Hyper-parameters. For OTDA and our method DPDA, we set the hyper-parameters $\lambda_{e}$ and $\lambda_{g}$ of Eq. (3) to 0.01 and 0.1 , respectively. In all benchmarks, we set the dimension of the subspace of our method $\ell=\frac{k}{10}$ and the noise-ratio $\frac{\sigma}{w}=1.1$. For the privacy budget, we again follow the standard of [Abadi et al., 2016; Papernot et al., 2017] by setting $\delta=\frac{1}{1.2 n_{s}}, \varepsilon=2$ for VisDA and $\varepsilon=8$ for the other datasets, except $\varepsilon=20$ if the source is DSLR or Webcam in Office-Caltech since they have too few samples (150-200 in total). For PATE and DPDA, we use the privacy accountant tool [Abadi et al., 2016] to keep track the privacy budget after each step as explained in Section 5.1. We run each test 3

\begin{tabular}{|c|cc|cc||cc|c|}
\hline & PATE & DPDA & OTDA & ADDA & t-only & DPDA & OTDA \\
\hline AC & 37.4 & 39.2 & 44.2 & 41.3 & 23.3 & 43.4 & 46.5 \\
AP & 52.1 & 54.4 & 58.5 & 56.5 & 56.8 & 64.2 & 65.2 \\
AR & 59.7 & 61.4 & 67.2 & 67.1 & 51.3 & 67.7 & 68.6 \\
CA & 48.7 & 50.3 & 55.3 & 49.6 & 41.9 & 55.8 & 57.2 \\
CP & 54.2 & 57.8 & 61.7 & 61.2 & 56.8 & 67.6 & 69.1 \\
CR & 56.7 & 59.9 & 64.0 & 63.2 & 51.3 & 63.5 & 66.4 \\
PA & 51.5 & 53.5 & 53.9 & 50.4 & 41.9 & 57.6 & 58.0 \\
PC & 36.8 & 40.7 & 42.9 & 45.4 & 23.3 & 45.8 & 46.1 \\
PR & 65.8 & 67.9 & 68.9 & 69.5 & 51.3 & 70.8 & 71.3 \\
RA & 57.6 & 59.4 & 61.6 & 58.8 & 41.9 & 63.1 & 63.6 \\
RC & 39.4 & 43.8 & 46.2 & 49.3 & 23.3 & 47.9 & 49.1 \\
RP & 66.2 & 68.7 & 70.1 & 70.9 & 56.8 & 72.8 & 73.5 \\
\hline Avr & 52.1 & 54.8 & 57.9 & 56.9 & 43.3 & 60.0 & 61.2 \\
\hline
\end{tabular}

Table 3: Performance (accuracy \%) on Office-Home dataset. Left: unsupervised, and right: semi-supervised.

times and report the average.

Results. The results are presented in Tables 1, 2 and 3. In both Office-Caltech and VisDA benchmarks, our model DPDA performs at the same level as non-private state-of-theart methods and even gets 1 point higher than ADDA in the VisDA benchmark, while PATE significantly lags behind. If we reduce the privacy budget for the source domains DSLR, Webcam in the Office-Caltech benchmark to $\varepsilon=10$, then the performances of DPDA and PATE drop by 3-5 points. In the Office-Home dataset, DPDA is 2-3 points lower than non-private baselines but still safely outperforms PATE by 3 points. On the other hand, in the semi-supervised scenario on the Office-Home benchmark, when only 1 labeled target sample per class is allowed, DPDA manages to reduce the gap with OTDA significantly from 3 points (55 vs 58 ) to 1 point (60 vs 61). This means that DPDA benefits more from some labeled target data than OTDA. Behind the good behavior of DPDA to deal with domain adaptation tasks, the results reported in these tables confirm that DPOT is a very performing differentially private optimal transport method.

\section{Conclusion}

In this paper, we have proposed the first differentially private approach for optimal transport. The proposed algorithm DPOT is able to preserve the Wasserstein distance despite the noisy perturbations introduced used to ensure differential privacy. We have then designed the first complete differentially private domain adaptation model based upon DPOT. Our methods are justified by strong theoretical guarantees and the experimental evaluations illustrated that our approach allows one to obtain good results while ensuring a high privacy level of the data. Future work includes extensions of our framework to other applications of optimal transport, for instance, in differentially private distributed learning.

\section{Acknowledgements}

Work supported by the ACADEMICS grant of the IDEXLYON, project of the Université de Lyon, PIA operated by ANR-16-IDEX-0005 


\section{References}

[Abadi et al., 2016] Martin Abadi, Andy Chu, Ian Goodfellow, H Brendan McMahan, Ilya Mironov, Kunal Talwar, and Li Zhang. Deep learning with differential privacy. In $C C S$, pages 308-318. ACM, 2016.

[Arjovsky et al., 2017] Martin Arjovsky, Soumith Chintala, and Léon Bottou. Wasserstein gan. arXiv:1701.07875, 2017.

[Balle et al., 2018] Borja Balle, Gilles Barthe, and Marco Gaboardi. Privacy amplification by subsampling: Tight analyses via couplings and divergences. In NIPS, pages 6280-6290, 2018.

[Blocki et al., 2012] Jeremiah Blocki, Avrim Blum, Anupam Datta, and Or Sheffet. The johnson-lindenstrauss transform itself preserves differential privacy. In FOCS, pages 410-419. IEEE, 2012.

[Chaudhuri and Monteleoni, 2009] Kamalika Chaudhuri and Claire Monteleoni. Privacy-preserving logistic regression. In NIPS, pages 289-296, 2009.

[Chollet, 2015] François Chollet. Keras. https://keras.io, 2015.

[Courty et al., 2017a] Nicolas Courty, Rémi Flamary, Amaury Habrard, and Alain Rakotomamonjy. Joint distribution optimal transportation for domain adaptation. In NIPS, pages 3730-3739, 2017.

[Courty et al., 2017b] Nicolas Courty, Rémi Flamary, Devis Tuia, and Alain Rakotomamonjy. Optimal transport for domain adaptation. IEEE TPAMI, 39(9):1853-1865, 2017.

[Cuturi, 2013] Marco Cuturi. Sinkhorn distances: Lightspeed computation of optimal transport. In NIPS, pages 2292-2300, 2013.

[Dwork et al., 2006] Cynthia Dwork, Frank McSherry, Kobbi Nissim, and Adam Smith. Calibrating noise to sensitivity in private data analysis. In TCC, pages 265-284. Springer, 2006.

[Dwork et al., 2010] Cynthia Dwork, Guy N Rothblum, and Salil Vadhan. Boosting and differential privacy. In FOCS, pages 5160. IEEE, 2010.

[Dwork et al., 2014] Cynthia Dwork, Aaron Roth, et al. The algorithmic foundations of differential privacy. Foundations and Trends $\AA$ in Theoretical Computer Science, 9(3-4):211-407, 2014.

[Flamary and Courty, 2017] R'emi Flamary and Nicolas Courty. Pot python optimal transport library, 2017.

[Frogner et al., 2015] Charlie Frogner, Chiyuan Zhang, Hossein Mobahi, Mauricio Araya, and Tomaso A Poggio. Learning with a wasserstein loss. In NIPS, pages 2053-2061, 2015.

[Ganin et al., 2016] Yaroslav Ganin, Evgeniya Ustinova, Hana Ajakan, Pascal Germain, Hugo Larochelle, François Laviolette, Mario Marchand, and Victor Lempitsky. Domain-adversarial training of neural networks. JMLR, 17(1):2096-2030, 2016.

[Guo et al., 2018] Xiawei Guo, Quanming Yao, WeiWei Tu, Yuqiang Chen, Wenyuan Dai, and Qiang Yang. Privacypreserving transfer learning for knowledge sharing. arXiv preprint arXiv:1811.09491, 2018.

[Hamm et al., 2016] Jihun Hamm, Yingjun Cao, and Mikhail Belkin. Learning privately from multiparty data. In $I C M L$, pages 555-563, 2016.

[Hardt and Roth, 2013] Moritz Hardt and Aaron Roth. Beyond worst-case analysis in private singular vector computation. In STOC, pages 331-340. ACM, 2013.

[Johnson and Lindenstrauss, 1984] William B Johnson and Joram Lindenstrauss. Extensions of lipschitz mappings into a hilbert space. Contemporary mathematics, 26(189-206):1, 1984.
[Kantorovich, 1942] Leonid Vitalievich Kantorovich. On the translocation of masses. In Dokl. Akad. Nauk. USSR (NS), volume 37, pages 199-201, 1942.

[Kenthapadi et al., 2013] Krishnaram Kenthapadi, Aleksandra Korolova, Ilya Mironov, and Nina Mishra. Privacy via the johnsonlindenstrauss transform. Journal of Privacy and Confidentiality, $5(1), 2013$.

[Pan and Yang, 2010] Sinno Jialin Pan and Qiang Yang. A survey on transfer learning. IEEE Transactions on knowledge and data engineering, 22(10):1345-1359, 2010.

[Papernot et al., 2017] Nicolas Papernot, Martín , Ulfar Erlingsson, Ian Goodfellow, and Kunal Talwar. Semi-supervised knowledge transfer for deep learning from private training data. In ICLR, 2017.

[Pathak et al., 2010] Manas Pathak, Shantanu Rane, and Bhiksha Raj. Multiparty differential privacy via aggregation of locally trained classifiers. In NIPS, pages 1876-1884, 2010.

[Peng et al., 2017] Xingchao Peng, Ben Usman, Neela Kaushik, Judy Hoffman, Dequan Wang, and Kate Saenko. Visda: The visual domain adaptation challenge. arXiv:1710.06924, 2017.

[Perrot et al., 2016] Michaël Perrot, Nicolas Courty, Rémi Flamary, and Amaury Habrard. Mapping estimation for discrete optimal transport. In NIPS, pages 4197-4205, 2016.

[Redko et al., 2017] Ievgen Redko, Amaury Habrard, and Marc Sebban. Theoretical analysis of domain adaptation with optimal transport. In ECML-PKDD, pages 737-753. Springer, 2017.

[Reich, 2013] Sebastian Reich. A nonparametric ensemble transform method for bayesian inference. SIAM Journal on Scientific Computing, 35(4):A2013-A2024, 2013.

[Rubinstein et al., 2009] Benjamin IP Rubinstein, Peter L Bartlett, Ling Huang, and Nina Taft. Learning in a large function space: Privacy-preserving mechanisms for svm learning. arXiv preprint arXiv:0911.5708, 2009.

[Saenko et al., 2010] Kate Saenko, Brian Kulis, Mario Fritz, and Trevor Darrell. Adapting visual category models to new domains. In ECCV, pages 213-226. Springer, 2010.

[Shen et al., 2018] Jian Shen, Yanru Qu, Weinan Zhang, and Yong Yu. Wasserstein distance guided representation learning for domain adaptation. In $A A A I, 2018$.

[Shokri and Shmatikov, 2015] Reza Shokri and Vitaly Shmatikov. Privacy-preserving deep learning. In CCS, pages 1310-1321. ACM, 2015.

[Tzeng et al., 2017] Eric Tzeng, Judy Hoffman, Kate Saenko, and Trevor Darrell. Adversarial discriminative domain adaptation. In CVPR, pages 7167-7176, 2017.

[Vempala, 2005] Santosh S Vempala. The random projection method, volume 65. American Mathematical Soc., 2005.

[Venkateswara et al., 2017] Hemanth Venkateswara, Jose Eusebio, Shayok Chakraborty, and Sethuraman Panchanathan. Deep hashing network for unsupervised domain adaptation. In CVPR, 2017.

[Villani, 2008] Cédric Villani. Optimal transport: old and new, volume 338. Springer Science \& Business Media, 2008.

[Wang et al., 2018] Yang Wang, Quanquan Gu, and Donald Brown. Differentially private hypothesis transfer learning. In $E C M L$ PKDD, pages 811-826. Springer, 2018.

[Zoph et al., 2018] Barret Zoph, Vijay Vasudevan, Jonathon Shlens, and Quoc V Le. Learning transferable architectures for scalable image recognition. In CVPR, pages 8697-8710, 2018. 